\title{
Adherence to Clinical Practice Guidelines during Antenatal Management of Gestational Diabetes Mellitus: An Integrative Review
}

\author{
Nisreen I. Salama ${ }^{1}$, Lubna Abushaikha ${ }^{2}$ \\ ${ }^{1}$ Arab American University, Jenin, Palestine \\ ${ }^{2}$ Maternal and Child Health Nursing Department, School of Nursing, The University of Jordan, Amman, Jordan \\ Email: *Nisreen.salama@aaup.edu
}

How to cite this paper: Salama, N.I. and Abushaikha, L. (2018) Adherence to Clinical Practice Guidelines during Antenatal Management of Gestational Diabetes Mellitus: An Integrative Review. Open Journal of Nursing, 8, 758-770.

https://doi.org/10.4236/ojn.2018.810057

Received: September 13, 2018

Accepted: October 27, 2018

Published: October 30, 2018

Copyright (c) 2018 by authors and Scientific Research Publishing Inc. This work is licensed under the Creative Commons Attribution International License (CC BY 4.0).

http://creativecommons.org/licenses/by/4.0/

\begin{abstract}
The number of pregnant women affected by gestational Diabetes (GDM) is increasing globally with an estimated prevalence as high as $15 \%$. Gestational Diabetes Miletus is associated with birth complications for women and newborn, includes development of type II diabetes, preeclampsia during pregnancy, increasing the risk of fetal loss, stillbirth and perinatal death. An integrative literature review applied, systematic search from different data base obtained from international and middle east countries to assess adherence level of guidelines. Clinical guidelines are set to ensure and assure homogeneity as well as the quality of provision of care. National and international consensus has yet to be achieved in the management of diabetes in pregnancy, adherence of recommended antenatal care content to a minimum level appears to be unmet. Complete provider adherence to first antenatal guidelines was $48.1 \%$, Guideline dissemination alone does not change practice; assessment of barriers/enablers and implementation is important. Guidelines are useless when they are not used or adhered to. Each guideline needs an Appendix on how adherence has to be measured, there is a need for some uniformity across guidelines to measure adherence. Diabetes management is an essential constituent to prevent prognosis of diabetes complications.
\end{abstract}

\section{Keywords}

Adherence, Antenatalcare, Clinical Practice Guidelines, Gestational Diabetes Mellitus

\section{Introduction}

Quality of care can be improved by decreasing unnecessary practice variation 
between professionals. One way to reduce practice variation is by moving evidence-based knowledge into daily practice. To facilitate the translation of the most recent evidence into practice, guidelines are developed and implemented [1].

Clinical guidelines are set to ensure and assure homogeneity as well as the quality of provision of care. Utilization of guidelines is influenced by many factors. Clinical practice guidelines are "statements that include recommendations intended to optimize patient care that is informed by a systematic review of evidence and an assessment of the benefit and harms of alternative care options". Guidelines contain practical evidence-based advice for professionals and patients and aim to improve the quality of care, guidelines are useless when they are not used or adhered to [1].

Adherence has been defined as "conformity to, accomplishing or following official or institutional requirements, guidelines, protocols, recommendations or standards" [2].

Antenatal care (ANC) is an effective measure that provides care to the pregnant women, quality is still a problem. Quality in maternal services involves providing a minimum level of care to all pregnant women, yet adherence of recommended ANC content to a minimum level appears to be unmet, adherence to clinical guideline is important especially in pre gestational complication like gestational diabetes mellitus [3].

Gestational Diabetes Mellitus (GDM) is defined as any degree of glucose intolerance with the onset or first recognition during pregnancy, and it is associated with several maternal and fetal effects includes development of type II diabetes, preeclampsia during pregnancy, increasing the risk of fetal loss, stillbirth and perinatal death [4] [5].

Globally GDM complicate about $1 \%-14 \%$ of all pregnancies, the prevalence rate in most European countries including United Kingdom is about 1\% - 2\% while among Asian women prevalence rate was higher nearly $11.9 \%$ especially Chinese and Indian women [6]. The prevalence of Diabetes in the Palestinian population in the West Bank, Gaza and E. Jerusalem is high around 15.3\%, average prevalence of GDM in Gaza was $1.8 \%$ and is increasing [6] [7].

The aim of this literature review was to critically analyze data from existing studies and explore the themes identified in relation to the adherence to clinical guideline during antenatal management of GDM, focus on assessment to adherence international and national level, the problem her according to literature non-adherence to clinical guidelines. Adherence has critical role in the determination of therapeutic outcomes and is considered a key factor in achieving therapeutic success. Therefore, a need to investigate this problem in our country exists. Guideline dissemination alone does not change practice; assessment of barriers/enablers an implementation design must be theory-driven [8].

\section{Materials and Methods}

In this review, the search was performed by different international databases in- 
cluding: Google Scholar, PubMed, Medline, CINAHL, EBSCO and the Jordanian Database for Nursing Research using the keywords of "adherence", "clinical practice guidelines", "antenatal care", "gestational diabetes mellitus", "prenatal care". These words were searched in combination with each other and in separated form. For example, during the initial search, the keyword "adherence" was combined with "clinical practice guideline". Later, during each new search a new keyword added until including all keywords in the last search. However, the searching process was limited to following inclusion criteria: full text, written in English, published between 2008 and 2018, and specifically related to adherence to clinical guidelines during GDM treatment in antenatal care.

However, review papers and incomplete reports in the form of editorials, opinion pieces, and conference abstracts have been excluded. Furthermore, a total of 1260 articles were retrieved and after removing the duplicated articles 900 articles remain, the initial evaluation for their titles abstracts took place, 579 excluded by title, 276 excluded by abstract, only 45 were found to be related to the topic of interest. Then, related articles were printed and read in full, following a secondary evaluation, 15 articles were exactly identified to cover the inclusion criteria. As a result, those fifteen articles were included in this review (Figure 1).

\section{Results}

Fifteen relevant articles have been reviewed. From each article the following data have been extracted: Title, author, study purpose, design, sitting and findings.

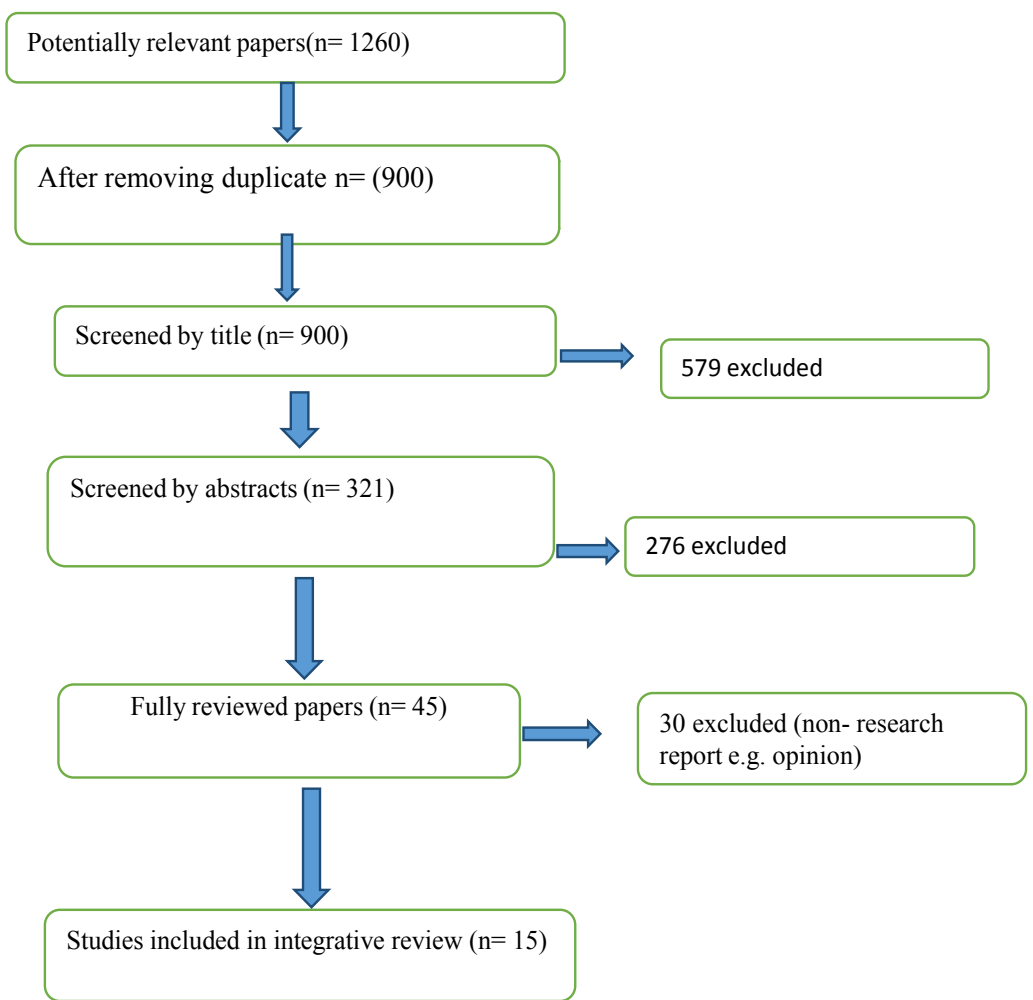

Figure 1. Search strategy and outcomes. PRISMA guideline. 
Then, the authors compared articles, interpreted findings to identify themes, and grouped them based on their similarities. Overall, the fifteen reviewed research articles published between 2008 and 2018 (three systematic review, eight cross sectionals quantitative study, one survey study and three descriptive). All articles presented in Appendix.

On the international level two study was conducted in Ghana, two study was conducted in Australia, one study was conducted in Malaysia, one study was conducted in each of Sweden, Netherlands, Korea. In the Middle East country one study was conducted in Riyadh, Saudi Arabia the other in Morocco, the third one in Palestine, the reaming studies was systematic review done in different countries. However, to address the purpose of this review, the findings (highlight adherence to clinical practice guidelines) were categorized into common themes, using a color-coding method to highlight similarities across the literature. After sorting and grouping the data, three thematic areas were identified these includes the following: adherence to clinical practice guidelines in GDM patient's; barriers and enablers, adherence to clinical GDM guidelines in studies from international countries, adherence to clinical GDM guidelines in studies from middle east countries.

\subsection{Theme One: Adherence to Clinical Practice Guidelines in GDM Patient's Barriers and Enablers}

Diabetes is a global health problem, in both developed and developing countries, the prevalence of gestational diabetes (GDM) in low and lower middle income countries (LLMIC) is increasing. Although its associated with short and long term complications for mothers and their newborns, there is a lack of knowledge about how to detect and manage GDM. Although, guidelines for GDM screening and management are available and freely downloadable from the internet from high income setting, there is a lack of knowledge about the degree to which guidelines developed in high income settings are appropriate foruse in low income settings or less developed countries, where resources are scarce and access to care is limited [9] [10].

Four studies about management of GDM in low and lower middle income country in Africa, South Asia, and Latin America. The results demonstrated high diversity in screening and management practices.

According to Utz, Kolsteren [11] they stated that management of GDM was found to take place mainly at the tertiary level, A cross-sectional survey was conducted between November 12, 2014 and May 11, 2015. Questionnaires were distributed to gynecologists, medical doctors and endocrinologists who were involved in providing care to patients with GDM. Questionnaires were sent to 182 individuals and 77 healthcare providers from 26 countries completed the survey. The results demonstrated high diversity in screening and management practices. Only $52(68 \%)$ participants reported that any guidelines were available in their setting. 
According to Utz, Delamou [12], there is a lack of knowledge on how the women with GDM are managed in low resource settings. A scoping review conducted of published literature, all articles containing information on clinical practices of detection and management of gestational diabetes published until April 24, 2016 included. Results: 23 articles identified mainly from Asia and sub-Saharan Africa. All studies show that lack of uniformity in the management of gestational diabetes requires a focus on the promotion of universal guidelines on GDM screening and management that are applicable to low resource settings.

\subsubsection{Barriers to Adherence}

According to Utz and De Brouwere [10], both providers and patients poses a substantial barrier to detection and management of GDM due to unavailability of guidelines combined with lack of knowledge about GDM, leading to deficiencies in screening and counseling. Additional expenses related to specific tests and changes in diet were identified as important challenges, limited access to regular monitoring and follow-up care as a result of distance and costs are also considered. Services were not available at all levels nor was adequate testing equipment. Lack of communication and collaboration between different specialists and treatment delays as a result of patients being seen by multiple providers.

According to [8], reasons for failure to adhere to best practice are multifactorial and include: unfamiliarity with Nutrition Practice Guidelines Among relevant staff; insufficient dietetic resources; and women's lack of awareness of the benefits of regular dietetic counselling. Barriers have been grouped into the theoretical domains of: Knowledge; Beliefs about consequences; Intentions; Social influences; Social/professional role and identity; Environmental context and resources; and Memory, attention and decision processes.

Four main categories of barriers to physician guideline adherence:

1) The payment system; Lack of uniformity leads to diffusion of the effects of Pay for Performance programs.

2) Lack of information technology systems; Insufficient access to guidelines at the point of care

3) Physician culture, beliefs and habits; physicians base clinical decisions on their personal experience, physicians generally believe that their own clinical practice is good

4) The guideline development process and utility and function, development process of guidelines presents their own obstacles to adherence, mainly:

a) The lack of sufficient flexibility and relevance to physician practice; and

b) The lack of transparency in guideline development [13].

\subsubsection{Enablers and Strategies to Adherence}

Adherence to guidelines is associated with better outcomes among patients with gestational diabetes. Enablers, identified through clinic observation and team discussion, project funding for dietetic clinic time, a positive research and audit culture of the organization. To improve adherence many strategies includes Engage physicians in guideline development and review process; Expedite new re- 
search to support guideline development; and Make guidelines actionable [8] [13].

According to previous studies guidelines dissemination alone does not change practice; assessment of barriers/enablers and implementation design must be theory-driven. Policy makers need to address these challenges by strengthening their health care system as a whole, relevant and effective strategies to apply guidelines in clinical practice are needed to improve patient outcomes.

\subsection{Theme Two: Adherence to Clinical GDM Guidelines in Studies from International Countries}

According [2] [14] guidelines are developed to ensure quality, uniformity and consistency of care for clients .Engagement of providers during the process of developing guidelines is important in ensuring that they adhere to the guidelines. There is discrepancy between provider knowledge and practice, especially in low and middle income settings, and reasons for this need to be explored. In a two study in Ghana, a cross-sectional analysis of a prospective cohort study on adherence to guidelines was carried out. Complete provider adherence to first antenatal guidelines was $48.1 \%$. Complete provider adherence to first antenatal visit guidelines is low across different facilitytypes in the Greater Accra region of Ghana, providers should be trained to adhere to the guidelines during provision of care to all pregnant women since adherence to the guidelines at first visit play a major role for the quality of care of the pregnant woman.

In Australia according to [8] [15] one study was conducted in two developed high-income countries to compare Austrian and Australian national guidelines for gestational and pre-gestational diabetes and evaluate the level to which physicians comply with their country's guideline. The other one to identify barriers and enablers that confront GDM management and treatment. When guidelines compared between Austrian (ÖDG, Austrian Diabetes Society) and Australian guidelines (ADIPS, Australasian Diabetes in Pregnancy Society), they show that National and international consensus has yet to be achieved in the management of diabetes in pregnancy.

Several studies was done by [3] [9] [16] [17] In Malaysia, Korea, Netherlands, Sweden ,all studies focus on adherence to clinical practice guidelines ,focus on adherence to antenatal guidelines during GDM care and outcomes in patients. Result show many different ways to measure adherence, adherence to guidelines varies widely. Adherence is positively related to: evidence base of guideline, practicality of guideline, automated support system, a positive attitude of professionals.

\subsection{Theme Three: Adherence to Clinical GDM Guidelines in Studies from Middle East Countries}

There is a lack of study about adherence to clinical practice guideline for treatment of GDM in middle east countries. Diabetes in the Middle East: over the past two decades, there had been changes in the lifestyle of eastern Mediterra- 
nean countries, this led to an increase in non-communicable diseases, such as hypertension, cardiovascular diseases and diabetes mellitus.

In a study done in Egypt, using cross-sectional design, the glycemic control and prevalence of microvascular and neuropathic complications were described among Egyptians with diagnosed diabetes. The results were; blindness 5\%, 21\% albuminuria, $42 \%$ had retinopathy, $22 \%$ neuropathy, clinical nephropathy prevalence was 7\%, foot ulcers were only 1\%. Other studies were made in Egypt and Jordan to measure the prevalence of obesity and related diabetes. The results from Egypt were; 16\% were obese and 4.9\% had diabetes [18].

Albarrak, Mohammed [19] conducted a study to assess diabetes care in primary clinics based on the guidelines of American Diabetes Association (ADA). A retrospective study of medical records and laboratory system was conducted between November 2011 and May 2012. In total, 200 diabetic patients from the database of Primary Care Clinics (PCCs) in King Khaled University Hospitals (KKUH) were randomly selected. Study revealed insufficiency of diabetes care, and proper guidelines are not followed in the care for diabetic patients in the PCCs in comparison to the ADA standards of care.

Utz, Assarag [20] conducted a study in Morocco to assess knowledge and practices of generalpractitioners, midwives and nurses working at primary health care facilities regarding screening and management of gestational diabetes (GDM). Antenatal care (ANC) attendance is high, first level health care providers, mainly nurses and midwives, are usually the first point of contact for pregnant women. Result show that public primary health care providers have a basic understanding of GDM but screening and management practices are not uniform. Most nurses and midwives lack pre-service training on gestational diabetes while $56.8 \%$ of the doctors had some pre-service training. After diagnosing GDM, $88.5 \%$ of providers refer patients to specialists, only $11.5 \%$ treat them as outpatients.

In Gaza strip in Palestine a study was conducted by Radwan, Elsous [21] to assess the level of good glycemic control, to examine factors influencing good glycemic control and to determine association between adherence to antidiabetic medications and glycosylated hemoglobin (HbAlc) .A cross-sectional design was employed among 369 patients with type 2 diabetes mellitus (T2DM). study showed that glycemic control was suboptimal. To improve glycemic control and prevent diabetes-related complications it's important to get Knowledge of factors associated with that were: older age, high medication adherence, and better health literacy.

\section{Discussion}

The current review showed that Gestational Diabetes Mellitus management is an essential constituent to prevent prognosis of GDM complications. The prevalence of gestational diabetes (GDM) in low and lower middle income countries (LLMIC) is increasing. These findings could provide valuable evidences to enhance concept of adherence to guidelines in international and middle east coun- 
tries for GDM women. The findings were clustered in three themes as following adherence to clinical practice guidelines in GDM patient's; barriers and enablers, adherence to clinical GDM guidelines in studies from international countries, adherence to clinical GDM guidelines in studies from middle east countries.

Fifteen studies conducted in the international level and middle east countries level to assess level of adherence to clinical practice guidelines in management and treatment of GDM, most of studies are cross sectional studies show no uniformity across guidelines so adherence more difficult to measure. Guidelines dissemination alone does not change practice, it's important to develop a monitoring system and specific method to measure adherence. Many barriers and challenges that face health care providers to adhere to clinical guidelines one of them Lack of resources, insufficient dietetic resources; and women's lack of awareness of the benefits of regular dietetic counselling, Physician culture, beliefs and habits.

Providers should be trained and supported to adhere to the guidelines during provision of care to all pregnant women.

No studies about adherence to clinical guidelines in Jordan or Palestine. In middle east countries in Morocco, Saudi Arabia two studies was conducted about Knowledge and practice related to gestational diabetes among primary health care providers, Evaluation of diabetes care management in primary clinics based on the guidelines of American Diabetes Association respectively. Further studies needed to assess adherence level of guidelines during antenatal care for GDM patients especially in developing countries.

\section{Conclusions}

To achieve a high quality of GDM management, national guidelines must be consistent and uniform; it must be integrated into local care processes. Adherence to clinical guidelines decrease prognosis of GDM complications. Complete provider adherence to first antenatal visit guidelines is low across different facility. Providers should be trained and supported to adhere to the guidelines during provision of care to all pregnant women.

An essential element in the success of the management plan is diabetes management standards. Most of the elements examined are not in full compliance with the ADA standard. Continues monitoring and self-review are recommended. There is a need of actions improving compliance to the local guidelines. Many barriers and enablers or facilitators to adherence present, we should consider it and try to overcome barriers in order to improve adherence to clinical guidelines and then improve quality of care and patient health.

\section{Acknowledgements}

Thank you to Prof. Muayyad Ahmad for reading an early copy of this paper.

\section{Conflicts of Interest}

The authors declare no conflicts of interest regarding the publication of this paper. 


\section{References}

[1] Ament, S.M.C., et al. (2015) Data from: Sustainability of Professionals' Adherence to Clinical Practice Guidelines in Medical Care: A Systematic Review. Dryad Digital Repository: Germany, Europe.

[2] Amoakoh-Coleman, M., et al. (2016) Client Factors Affect Provider Adherence to Clinical Guidelines during First Antenatal Care. PLoS ONE, 6.

[3] Yeoh, P.L., et al. (2015) Assessing the Extent of Adherence to the Recommended Antenatal Care Content in Malaysia: Room for Improvement. Plos One, 10, e0135301-e0135301. https://doi.org/10.1371/journal.pone.0135301

[4] Gillespie, P., et al. (2013) Modeling the Independent Effects of Gestational Diabetes Mellitus on Maternity Care and Costs. Diabetes Care, 36, 1111-1116. https://doi.org/10.2337/dc12-0461

[5] Rani, P.R. and Begum, J. (2016) Screening and Diagnosis of Gestational Diabetes Mellitus, Where Do We Stand. Journal of Clinical and Diagnostic Research, 10, Qe01-4. https://doi.org/10.7860/JCDR/2016/17588.7689

[6] Alkasseh, A., et al. (2014) Prevalence and Associated Demographic Characteristics of Gestational Diabetes Mellitus in Gaza. Health and the Environment Journal, 5, $10-25$.

[7] Anders, D., et al. (2017) Palestine National Diabetes Program, WDF15-1304. https://www.worlddiabetesfoundation.org/projects/west-bank-and-gaza-wdf15-130 4\#

[8] Wilkinson, S.A., et al. (2014) Barriers and Enablers to Translating Gestational Diabetes Guidelines into Practice. Practical Diabetes, 31, 67-72a.

https://doi.org/10.1002/pdi.1833

[9] Oh, S.-W., et al. (2011) Adherence to Clinical Practice Guidelines and Outcomes in Diabetic Patients. International Journal for Quality in Health Care: Journal of the International Society for Quality in Health Care, 23, 413-419. https://doi.org/10.1093/intqhc/mzr036

[10] Utz, B. and De Brouwere, V. (2016) Why Screen if We Cannot Follow-Up and Manage? Challenges for Gestational Diabetes Screening and Management in Low and Lower-Middle Income Countries: Results of a Cross-Sectional Survey. BMC Pregnancy \& Childbirth, 16, 1-8. https://doi.org/10.1186/s12884-016-1143-1

[11] Utz, B., Kolsteren, P. and De Brouwere, V. (2016) Clinical Article: A Snapshot of Current Gestational Diabetes Management Practices from 26 Low-Income and Lower-Middle-Income Countries. International Journal of Gynecology and Obstetrics, 134, 145-150. https://doi.org/10.1016/j.ijgo.2016.01.020

[12] Utz, B., et al. (2016) Detection and Management of Diabetes during Pregnancy in Low Resource Settings: Insights into Past and Present Clinical Practices. Journal of Diabetes Research, 2016, 3217098-3217098. https://doi.org/10.1155/2016/3217098

[13] Kenefick, H., Lee, J. and Fleishman, V. (2008) Improving Physician Adherence to Clinical Practice Guidelines, Barriers and Strategies for Change. New England Healthcare Institute.

[14] Amoakoh-Coleman, M., et al. (2016) Public Health Facility Resource Availability and Provider Adherence to First Antenatal Guidelines in a Low Resource Setting in Accra, Ghana. BMC Health Services Research, 1.

[15] Zeck, W., et al. (2007) Management of Diabetes in Pregnancy: Comparison of Guidelines with Current Practice at Austrian and Australian Obstetric Center. Croatian Medical Journal, 48, 831-841. https://doi.org/10.3325/cmj.2007.6.831 
[16] Mistiaen, P., et al. (2012) Guideline Adherence in the Netherlands: A Systematic Review. German Medical Science GMS Publishing House, Düsseldorf.

[17] Persson, M., Winkvist, A. and Mogren, I. (2009) Surprisingly Low Compliance to Local Guidelines for Risk Factor Based Screening for Gestational Diabetes Mellitus-A Population-Based Study. BMC Pregnancy and Childbirth, 9, 53.

https://doi.org/10.1186/1471-2393-9-53

[18] Jilleh, C. (2013) The Interaction between Health Service Providers and People with Diabetes in Palestine.

[19] Albarrak, A.I., et al. (2018) Evaluation of Diabetes Care Management in Primary Clinics Based on the Guidelines of American Diabetes Association. Journal of Health Sciences, 12, 40-44.

[20] Utz, B., et al. (2017) Knowledge and Practice Related to Gestational Diabetes among Primary Health Care Providers in Morocco: Potential for a Defragmentation of Care? Primary Care Diabetes, 11, 389-396. https://doi.org/10.1016/j.pcd.2017.04.005

[21] Radwan, M., et al. (2017) Glycemic Control among Primary Care Patients with Type 2 Diabetes Mellitus in the Gaza Strip, Palestine. Therapeutic Advances in Endocrinology and Metabolism, 9, 3-14. 


\section{Appendix}

\begin{tabular}{|c|c|c|c|c|c|}
\hline Title & Author(s) & Purpose & A design & Sitting & Result \\
\hline
\end{tabular}

Evaluation of diabetes care management in primary clinics based on the guidelines of American Diabetes Association
Public health facility resource availability and provider adherence to first antenatal guidelines in a low resource setting in Accra, Ghana

Client Factors Affect Provider Adherence to Clinical Guidelines during First Antenatal Care

Knowledge and practice related to gestational diabetes among primary health care providers in Morocco: Potential for a defragmentation of care?

"Why screen if we cannot follow-up and manage?" Challenges for gestational diabetes screening and management in low and lower-middle income countries: results of a cross-sectional survey

Detection and

Management of Diabetes during Pregnancy in Low Resource Settings: Insights into Past and Present Clinical Practices

The main objective of this study was to assess diabetes care in primary A retrospective study at

Albarrak, Mohammed [19] clinics based on the guidelines of American King Khaled University Diabetes

Association (ADA).

The result showed that elements achieving the ADA targets for overall care were medical history (44.9\%), physical examination (59.6\%), laboratorye valuation (36.3\%), and referrals (19.3\%). The other subelement

Riyadh, Saudi Arabia. indicators such asreferral to diabetes self-management education clinics (10\%), dental examination (2\%), HbAlc regular monitoring (33.5\%), and blood pressure determination (100\%) were documented with adherence to ADA standards.

Eleven facilities comprising 6 hospitals (54.5\%), 4 polyclinics (36.4\%) and 1 health center were randomly sampled. Complete public health facility

A cross-sectional analysis of the baseline data of a Amoakoh-Coleman, resource availability in prospective cohort study Agyepong [14] relation to provider adherence to first was carried out in 11 facilities in the Greater antenatal visit guidelines. Accra Region of Ghana.
Our objectives were to determine the level of provider adherence to first antenatal visit

Amoakoh-Coleman, guidelines in the Safe Agyepong [2] Motherhood Protocol (SMP), and assess patient factors that determine complete provider adherence.

The objective of this study was to assess knowledge and practices Structured interviews with of general practitioners, 100 doctors, midwives and nurses and midwives nurses at 44 randomly working at primary Selected public health Care health care facilities in centers were conducted in Morocco regarding screening and management of gestational diabetes (GDM). Marrakech and $\mathrm{Al} \mathrm{Haouz.}$ All data Were descriptively analyzed. of a cohort study that recruited women who delivered in eleven health facilities and who had utilized antenatal care services during their pregnancy in the Greater Accra region of Ghana

Utz, Assarag [20]
Ghana. provider adherence to first antenatal guidelines for all the 946 participants was $48.1 \%$ varying significantly amongst the types of facilities, with highest rate in the polyclinics.

Cross-sectional study is part

The objective of our study was to identify the

Utz and De Brouwere [10] challenges that first line healthcare providers in LLMIC face in screening and management of GDM.

We conducted a cross-sectional survey of key informants from 40 low and lower-middle income by sending out questionnaires to 182 gynecologists, endocrinologists and medical doctors

To identify modalities of a scoping review was gestational diabetes conducted of published detection and literature and searched the management in low and databases all articles lower middle income countries. published until April 24 2016 included.
Complete adherence to guidelines pertained to only $48.1 \%$ of pregnant women. Providers were significantly more likely to completely

Ghana. adhere to guidelines when caring for multiparous women but less likely to do so when attending to women with history of previous pregnancy complications

Public primary health care providers have a basic understanding of gestational diabetes but screening and management practices are

Morocco: not uniform. Although 56.8\% of the Doctors had some pre-service training on gestational diabetes, most nurses and midwives lack such training

Unavailability of guidelines combined with lack of knowledge about GDM on the part of

Africa, both providers and patients poses a

South-Asia and $\begin{aligned} & \text { both providers and patients poses } \\ & \text { substantial barrier to detection and }\end{aligned}$ Latin-America management of GDM, leading to deficiencies in screening and counseling.

23 articles were identified mainly from Asia Asia and and sub-Saharan Africa. The majority of sub-Saharan studies were conducted in large tertiary care Africa. centers and hospital admission was reported in a third of publications. 
Clinical article: A snapshot of current gestational diabetes management practices from 26 low-income and

lower-middle-income countrie
Utz, Kolsteren [11] meelitu (GDM) in low-income and lower-middle-income countries.
Barriers and enablers to translating gestational diabetes guidelines into practice

Surprisingly low compliance to local guidelines for risk factor based screening for gestational diabetes mellitus-A

population-based study

Assessing the Extent of Adherence to the Recommended Antenatal Care Content in Malaysia: Room for

Improvement

\section{Management of} diabetes in pregnancy: comparison of guidelines with current Zeck, Panzitt [15] practice at Austrian and Australian obstetric center

Guideline adherence in the Netherlands: a systematic review

Sustainability of professionals' adherence to clinical practice guidelines in medical care: a systematic review

A cross-sectional survey was

conducted between

November 12, 2014 and

To identify screening and May 11, 2015 management practices for

Questionnaires were distributed to who were involved in providing care to patients with GDM in

low-income or

lower-middle-income countries in Africa, South Asia, and Latin America.

the outline recommended by French et al. that

Analyze Barriers and Wilkinson, McCray enablers to translating [8] gestational diabetes guidelines into practice involved four steps to assess influencing factors and design of implementation Australia strategies in a translational research project was followed

Africa, South countries completed the survey. The results Asia, and Latin demonstrated high diversity in screening and America. management practices. Only 52 (68\%) participants reported that any guidelines were available in their setting.

Of 44 clinic staff surveyed, most believed regular dietetic contact could influence diet, but fewer believed contact could influence blood glucose level (BGLs), pharmacotherapy, and care costs, and only half felt contact could influence weight gain or macrosomia

To investigate: 1) the compliance with local guidelines of screening for GDM and 2) the

Persson, Winkvist outcomes of pregnancy

population-based retrospective cross-sectional study of 822 women. A combination of questionnaire data and data collected from medical records was applied. [17] and birth in relation to and birth in relation to whether or not exposed to oral glucose tolerance test (OGTT).

Analyze adherence to recommended antenatal care (ANC); specifically, to examine the extent of

Yeoh, Hornetz [3] adherence to

recommended ANC

content and to determine of 522 randomly selected women who used ANC was Malaysia conducted.

the factors influencing ANC content score.

Sweden

Austrian (ODG, Austrian

To compare Austrian and Diabetes Society) and Australian national Australian guidelines guidelines for gestational (ADIPS, Australasian and pre-gestational Diabetes in Pregnancy level to which physicians gestational diabetes and comply with their country's guidelines. pre-gestational diabetes were systematically reviewed.

To investigate the adherence rate to Dutch

[16] guidelines, the influential Systematic Review factors to it and the effects of it

Netherlands

To evaluate 1) the state of

the art in sustainability research

[1] and 2) the outcomes of Systematic Review professionals recommendations in medical practice adherence:

1) Health care professional

2) Guideline

3) Organization/system

4) Implementation

5) Patient

More variation was found in the management of gestational than pre-gestational diabetes. There were differences in oral glucose diagnosing gestational diabetes in both centers and guidelines.

The search retrieved 4219 items, of which 14 studies met the inclusion criteria, involving 18 sustainability evaluations. 


\section{Continued}

Adherence to clinical practice guidelines and outcomes in diabetic patients

Glycemic control among primary care patients with type 2 diabetes mellitus in the Gaza Strip, Palestine
To examine the level of

adherence to clinical

practice guidelines and its

[9] relationship to outcomes

Retrospective cohort study in patients with diabetes.

to assess the level of good

glycemic control, to

determine association

between adherence to

A cross-sectional design was

[21] antidiabetic medications

and glycosylated

employed among $369 \quad$ Gaza Strip, patients with type 2 diabetes

(

to examine factors

influencing good

glycemic control.
Testing rates for blood pressure, eye exami-

nation, $\mathrm{HbA} 1 \mathrm{c}$, renal function and lipid

profiles were $93.9 \%, 32.8 \%, 84.9 \%, 33.5 \%$ and

$45.9 \%$, respectively. The adherence to

recommendations was unsatisfactory.

Mean of HbAlc was 8.97 (2.02) and one fifth of patients had good glycemic control

Strip, $\quad(\mathrm{HbA} 1 \mathrm{c} \leq 7 \%)$. Factors associated with good glycemic control were: older age, high medication adherence and better health literacy 